\title{
2D and 3D Shape Based Segmentation Using Deformable Models
}

\author{
Ayman El-Baz ${ }^{1}$, Seniha E. Yuksel ${ }^{1}$, Hongjian Shi ${ }^{1}$, Aly A. Farag ${ }^{1}$, \\ Mohamed A. El-Ghar ${ }^{2}$, Tarek Eldiasty ${ }^{2}$, and Mohamed A. Ghoneim² \\ 1 Computer Vision and Image Processing Laboratory, \\ University of Louisville, Louisville, KY 40292 \\ farag@cvip.louisville.edu \\ http://www.cvip.louisville.edu \\ 2 Mansoura University, Urology and Nephrology Center, Mansoura, Egypt
}

\begin{abstract}
A novel shape based segmentation approach is proposed by modifying the external energy component of a deformable model. The proposed external energy component depends not only on the gray level of the images but also on the shape information which is obtained from the signed distance maps of objects in a given data set. The gray level distribution and the signed distance map of the points inside and outside the object of interest are accurately estimated by modelling the empirical density function with a linear combination of discrete Gaussians (LCDG) with positive and negative components. Experimental results on the segmentation of the kidneys from low-contrast DCE-MRI and on the segmentation of the ventricles from brain MRI's show how the approach is accurate in segmenting $2-\mathrm{D}$ and $3-\mathrm{D}$ data sets. The $2 \mathrm{D}$ results for the kidney segmentation have been validated by a radiologist and the $3 \mathrm{D}$ results of the ventricle segmentation have been validated with a geometrical phantom.
\end{abstract}

\section{Introduction}

Both parametric deformable models and geometrical deformable models (level sets) are powerful methods and have been used widely for the segmentation problems; however, they both tend to fail in the case of noise, poor image resolution, diffused boundaries or occluded shapes, and they don't take advantage of the a priori models. Yet, especially in the area of medical imaging, organs have well constrained forms within a family of shapes [1. Thus, additional constraints based on the shape of the objects are greatly needed besides the gray level information of these objects.

To allow shape driven segmentation, Leventon et.al. [2] used a shape prior whose variance is obtained thorough PCA, and used this shape prior to evolve the level sets to the maximum a posteriori shape. Chen et al. 3. defined an energy functional which basically minimizes an Euclidean distance between a given point and its shape prior. In [4, a representation of the segmenting curve was generated based on the pose and shape parameters of a training set, which were optimized using a region based energy functional. In [15] a shape prior and its variance 
obtained from training data are used to define a Gaussian distribution, which is then used in the external energy component of a level sets framework. In [6], a shape boundary prior was formed from the features of the boundary, and this boundary was used in a level set framework. Recently, Tsai et.al. 7] used a deterministic model to represent the desired shape as a linear combination of weighted signed 2D distance maps and estimated these weights by minimizing a mutual information based cost function. And Yang et.al. 8 described the shapes with a multidimensional Gaussian probability model of these weights.

Different from the previous studies, in our approach, instead of directly using the average image itself, we are estimating the density of the signed distance map inside and outside the object of interest of the registered training samples by our modified EM algorithm and use this estimated density in the external energy component of the proposed deformable model framework. This representation of shapes is invariant to rotation and translation, and it overcomes the deformable models' inability to stop in high noise or in the case of missing edges.

\section{Proposed Deformable Models}

In conventional deformable models, surfaces move in the direction that minimizes an energy function given as [9]:

$$
E=E_{\text {int }}+E_{\text {ext }}=\int_{\tau \in T}\left(\xi_{\text {int }}(\phi(\tau))+\xi_{\text {ext }}(\phi(\tau))\right) d \tau
$$

where $\xi_{\text {int }}(\phi(\tau))$ and $\xi_{\text {ext }}(\phi(\tau))$ denote the internal and external forces respectively.

Typical external forces designed in [9] lead a deformable model toward edges in a $2 \mathrm{D} / 3 \mathrm{D}$ grayscale image. This and the other traditional external forces (e.g. based on lines or, edges, or the gradient vector flow) fail to make the deformable model closely approach an intricate boundary with concavities. Moreover, due to high computational complexity, the deformable models with such external energies are slow compared to the other segmentation techniques.

As a solution to these problems, we modify the external energy component of this energy formulation, and we formulate an energy function using the density estimations of two distributions: the signed distance map from shape models and the gray level distribution. The external energy component of our deformable models is formulated as:

$$
\xi_{\text {ext }}(\phi(\tau))=\left\{\begin{array}{r}
-p_{g}(q \mid k) p_{s}(d \mid k) \text { if } k=k^{*} \\
p_{g}(q \mid k) p_{s}(d \mid k) \text { if } k \neq k^{*}
\end{array}\right.
$$

In this formulation, $k$ is the region label with $k=1, \ldots, K, q$ is the gray level and $d$ is the signed distance where $p_{s}(d \mid k)$ is the density that describes the signed distance map inside and outside the object, and $p_{g}(q \mid k)$ is the density estimation of the gray level. With this energy function, the stochastic external force for each control point $\phi(\tau)$ of the current deformable model evolves in a region $k^{*}$. 
Specifically for the examples to be given in section 3 we assume that the empirical density comes from two classes, i.e. $k=1,2$. For the kidney segmentation problem, these classes are the kidney and the other tissues, and for the ventricle segmentation, the first class is the gray matter, white matter, fat and bones; while the second class is the CSF of the brain (inside and outside the ventricles).

\subsection{Shape Model Construction}

The signed distance map density $p_{s}(d \mid k)$ in the above mentioned external energy is calculated using a shape model, which is basically an average surface shape obtained from the images in the data set. The steps to construct this average surface shape is as follows:

1. Align the images in the data set using $2 \mathrm{D} / 3 \mathrm{D}$ rigid registration based on Mutual Information 10.

2. Manually segment the objects of interest from the database.

3. Calculate the $2 \mathrm{D} / 3 \mathrm{D}$ edge $V$ that describes the boundary of the object for all the manually segmented $\mathrm{N}$ number of images obtained in Step 2.

4. From the $\mathrm{N}$ number of shapes calculated in Step 3, find the average 2D/3D shape $V_{m}$ of the object by: $\quad V_{m}=\frac{1}{N} \sum_{i=1}^{N} V i$.

5. From the average shape, the distribution of the signed distance map inside and outside the 3D shape is calculated as follows: $(z=0$ for $2 \mathrm{D})$

$$
d(x, y, z)=\left\{\begin{array}{cc}
0 & (x, y, z) \in V \\
S((x, y, z), V) & (x, y, z) \in R_{V} \\
-S((x, y, z), V) & \text { Otherwise }
\end{array}\right.
$$

where $R_{v}$ is the region lying inside the shape and $S((x, y, z), V)$ is the minimum Euclidean distance between the image location $(x, y, z)$ and the curve $V$. This shape model is constructed only once throughout the whole algorithm.

The results of the average shape and signed distance calculations are shown for the kidney in Fig. 2, and the density estimation of this average shape is calculated using our modified EM approach which will be explained in Sec. 2.2

\subsection{Density Estimation}

In this paper we will use our modified Expectation-Maximization algorithm that approximates an empirical probability density function of scalar data with a linear combination of discrete Gaussians (LCDG) with positive and negative components.

In the following, we describe this model to estimate the marginal density for the gray level distribution $p_{g}(q)$ in each region. The same approach is also used to estimate the density of the signed distances $p_{s}(d)$ in the given image.

Let $q \in \mathbf{Q}=\{0,1, \ldots, Q-1\}$ denote the $Q$-ary gray level. The discrete Gaussian (DG) is defined as the discrete probability distribution $\Psi_{\theta}=(\psi(q \mid \theta)$ : $q \in \mathbf{Q})$ on $\mathbf{Q}$ such that $\psi(q \mid \theta)=\Phi_{\theta}(q+0.5)-\Phi_{\theta}(q-0.5)$ for $q=1, \ldots, Q-2$, 
$\psi(0 \mid \theta)=\Phi_{\theta}(0.5), \psi(Q-1 \mid \theta)=1-\Phi_{\theta}(Q-1.5)$ where $\Phi_{\theta}(q)$ is the cumulative Gaussian (normal) probability function with a shorthand notation $\theta=\left(\mu, \sigma^{2}\right)$ for its mean, $\mu$, and variance, $\sigma^{2}$.

In contrast to a conventional mixture of Gaussians and/or other simple distributions, one per region, we closely approximate the empirical gray level distribution for the given image with a LCDG having $C_{\mathrm{p}}$ positive and $C_{\mathrm{n}}$ negative components:

$$
p_{g: \mathbf{w}, \boldsymbol{\Theta}}(q)=\sum_{r=1}^{C_{\mathrm{p}}} w_{\mathrm{p}, r} \psi\left(q \mid \theta_{\mathrm{p}, r}\right)-\sum_{l=1}^{C_{\mathrm{n}}} w_{\mathrm{n}, l} \psi\left(q \mid \theta_{\mathrm{n}, l}\right)
$$

under the obvious restriction on the positive weights $\mathbf{w}=\left[w_{p, .}, w_{n, .}\right]$ :

$$
\sum_{r=1}^{C_{\mathrm{p}}} w_{\mathrm{p}, r}-\sum_{l=1}^{C_{\mathrm{n}}} w_{\mathrm{n}, l}=1
$$

To estimate the parameters for the model shown in Eq. (4), we will use our modified EM algorithm that modified the conventional EM algorithm to take into account both positive and negative discrete Gaussian components. The details of the algorithm are shown in [11.

\subsection{Stepwise Deformable Model Algorithm}

For any given image, the proposed algorithm of segmenting the region $k^{*}$ is as follows:

1. Register the given image to an average image using Mutual Information [10, where the average image is obtained by averaging the registered images of the database of Step 1 in Sec. 2. This step makes the algorithm invariant to scaling, rotation and translation.

2. Use the modified EM algorithm to estimate the density for signed distance map $p_{s}(d \mid k)$ inside and outside the object of interest from the average shape which was calculated a priori.

3. Calculate the normalized histogram for the given image/volume.

4. Use the modified EM algorithm to estimate the density for each class $p_{g}(q \mid k)$, $k$ being the class number $k=1 \ldots K$.

5. Initialize the control points $\phi(\tau)$ for the deformable model, and for each control point $\phi(\tau)$ on the current deformable model, calculate sign distances indicating exterior $(-)$ or interior $(+)$ positions of each of the eight nearest neighbors w.r.t. the contour.

6. Check the label $k=\mathbf{X}(\phi(\tau))$ for each control point:

(a) If the point is assigned to the region $k=k^{*}$, then

i. Estimate the region labels for its neighbors using Bayesian classifier such that they have the $(-)$ distance.

ii. If some of these sites are also assigned to the class $k^{*}$, then move the control point to the neighboring position ensuring the minimum total energy (i.e., expand the contour).

iii. Otherwise, do not move this point (the steady state). 
(b) If the point is assigned to the region $k \neq k^{*}$, then

i. Estimate the region labels for its neighbors using Bayesian classifier such that they have the $(+)$ distance.

ii. Move the control point to the neighboring position ensuring the minimum total energy (i.e. contract the contour)

7. If the iteration adds new control points, use the cubic spline interpolation of the whole surface and then smooth all its control points with a low pass filter.

8. Repeat steps [5 [6] and 7 until no positional changes occur in the control points.

\section{Experimental Results}

This section presents the results of our approach to segment the kidneys from the Dynamic Contrast-Enhanced MR images of the abdomen in 2D, and to segment the brain ventricles from MR images in $3 \mathrm{D}$.

\subsection{D Results for the Kidney}

In this study, Dynamic Contrast-Enhanced MRI (DCE-MRI), has been employed by a Signa Horizon GE 1.5T scanner using a contrast agent Gadolinium DTPA. In DCE-MRI, after the injection of a contrast agent Gd-DTPA, the abdomen is scanned rapidly and repeatedly resulting in high noise images because of the fast image acquisition. Moreover, the contrast of the kidney in the images changes continuously as the contrast agent perfuses into the kidney, resulting in very low contrast at some stages of the perfusion, necessitating the use of shape models in segmentation.

A typical DCE-MRI scan of a kidney is given in Fig. 1(a) with its empirical density (normalized histogram) shown in blue and the estimation of this density shown in red in Fig. 1(b). Figure 1(c) shows the positive and negative LCDG components used in our modified EM algorithm, and Fig. 1 (d) shows the estimated density for each class.

In Fig. 2(a) the average shape of a kidney obtained from 50 subjects is given with the signed distance map inside and outside the object as in Fig. 2(b). The density of this signed distance map is estimated with the same density estimation approach, the results of which are shown in Fig. 2(c), (d).

Finally, Fig. 3 shows the segmentation results of our approach compared to the segmentations by a radiologist.

\subsection{D Results for the Brain Ventricles}

In the following part, we use the proposed segmentation approach on $3 \mathrm{D}$ data sets collected from 20 subjects to segment the lateral ventricles of the brain in MR images, and in the second part, we are evaluating the segmentation approach using the cone-beam CT scans of a mould ventricle phantom. 


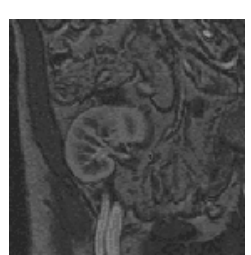

(a)

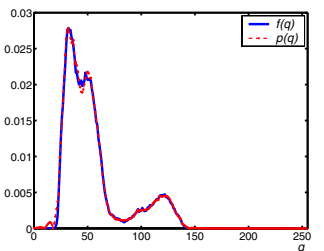

(b)

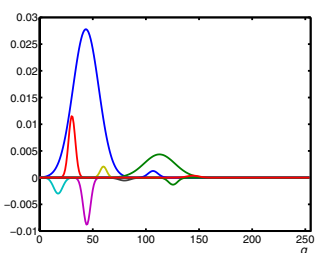

(c)

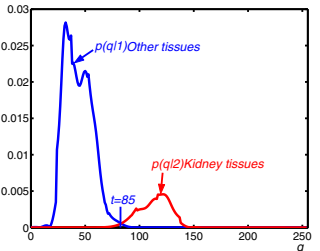

(d)

Fig. 1. A typical MRI scan of a kidney (a), and its gray level density estimation with the Modified EM Algorithm: (b) LCG components of the density estimation, (c) The final estimated density $p_{g}(q)$ for the empirical density $f(q)$ of the kidney image, (d) The marginal density estimation for each class.

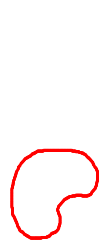

(a)

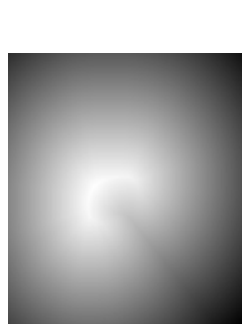

(b)



(c)

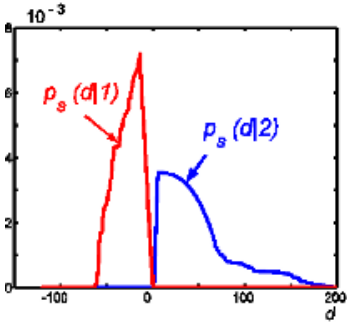

(d)

Fig. 2. (a) An average shape for a certain cross-sections of the kidney. (b) The signed distance map of this shape. (c) Density estimation for the signed distance. (d) Marginal density estimations for each class.

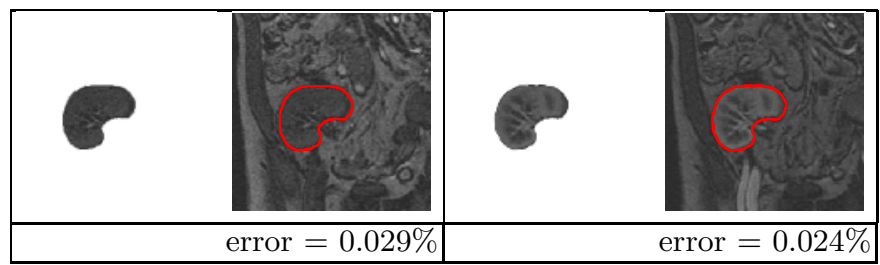

Fig. 3. Segmentation results (shown in red to the right) w.r.t. the radiologist's segmentation (to the left).

For ventricle segmentation from MRI, twenty data sets are not enough to get an accurate shape for the ventricles because the ventricles vibrate during the MRI or CT scans. Therefore, to cover all the shape variations of the brain ventricles for each subject, we performed finite element analysis on the motion of the real brain ventricles. For finite element analysis of the ventricle shape changes, assuming the cerebrospinal fluid (CSF) inside lateral ventricles is isotropic and linear elastic, we employed the linear elastic mechanical model. The Young's modulus of the CSF is 1000 Pascals and the Poisson ratio is 0.499 ([12]). For 


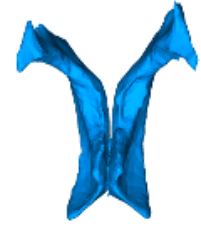

(a)

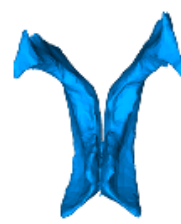

(b)

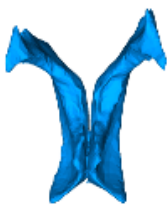

(c)

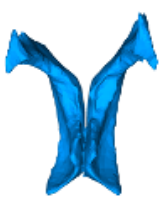

(d)

Fig. 4. The four states of the real ventricles at $t=0,0.7,0.9,1.1$ seconds (final) from left to right.

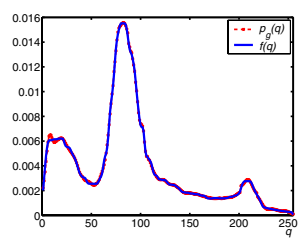

(a)

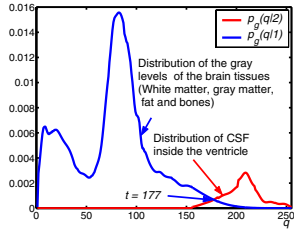

(b)

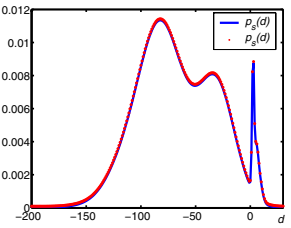

(c)

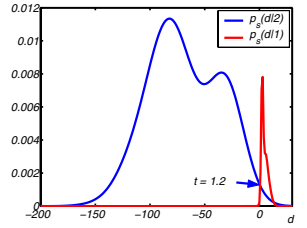

(d)

Fig. 5. (a) Final density estimation for the mixed frequency gray level distribution, (b) The class model of gray level for each class, (c) Final density estimation for the sign map inside and outside the 3-D brain ventricle, (d) The final class model of signed distance map for each class.

adults, the pressure of the CSF under normal conditions ranges between 50 and $180 \mathrm{mmH}_{2} \mathrm{O}$ with a median pressure of $115 \mathrm{mmH}_{2} \mathrm{O}$. Therefore, the uniform pressure of $115 \mathrm{mmH}_{2} \mathrm{O}$ is applied over the surfaces of the ventricles for each subject, and finite element analysis is performed to capture the variation of the ventricles. After finite element analysis, the $3 \mathrm{D}$ structure is re-sliced and 10 states of the ventricles are obtained resulting in 20 subjects $\times 10$ states $=200$ datasets. The four states of one subject's ventricles are shown in Fig. 4. Using the resulting 200 data sets for the ventricles, we followed our density estimation approach to estimate the density of the gray level distribution and the signed distance map inside and outside the ventricles. The results of density estimation using the proposed approach are shown in Fig. 5 and the segmentation results at different signal to ratios (SNR) (obtained by adding Gaussian noise with different variance) are shown in Fig. 6.

The hand segmentation of the radiologists may not always be accurate because of hand shaking. Therefore, to get accurate evaluation of our proposed approach, we used a mould ventricle phantom that resembles the geometrical structure of the real ventricles, scanned it with cone-beam CT, and used the scans for finite element simulation. Following the same procedure of the ventricle motion estimation, we captured all variations of the phantom ventricles. For the database obtained from the geometrical phantom, the inverse mapping method was used to get the same gray level distribution with the real ventricles; the gray level distribution of which was shown in Fig. 5(b). The final step of our algorithm is to estimate the pdf that describes the signed distance map inside 


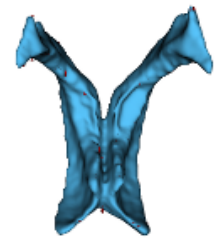

(a)

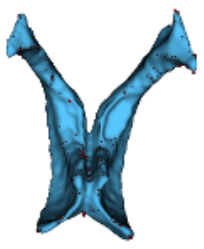

(b)

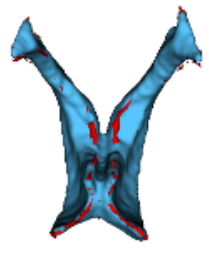

(c)

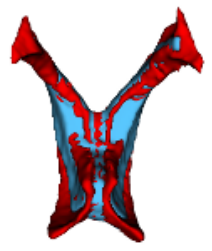

(d)

Fig. 6. (a) Results of our segmentation approach at SNR $=28 \mathrm{~dB}$, error $=0.01 \%$ (b)at $\mathrm{SNR}=7.8 \mathrm{~dB}$, error $=0.2 \%$ (c)at $\mathrm{SNR}=-1.9 \mathrm{~dB}$, error $=2.8 \%$. (d) Result of the segmentation errors using the conventional EM algorithm as a density estimator at $\mathrm{SNR}=28 \mathrm{~dB}$, error $=18.8 \%$. The errors are shown in red.

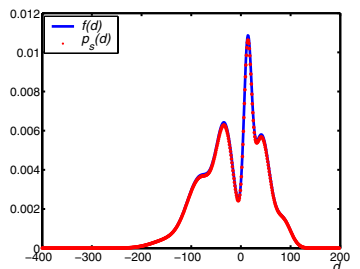

(a)

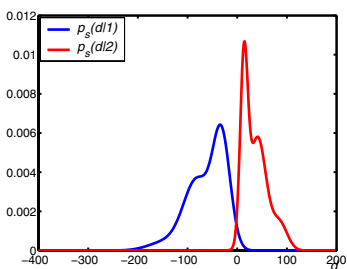

(b)

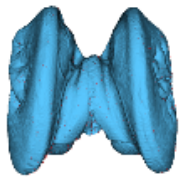

(c)

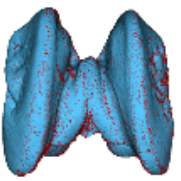

(d)

Fig. 7. (a) Final density estimation for the signed distance map inside and outside the 3 -D ventricle phantom, (b) The final class model of signed distance map for each class. Segmentation using our approach at different SNR (c) $S N R=28 d B$, error $=0.008 \%$, (d) $S N R=-1.9 \mathrm{~dB}$, error $=1.76 \%$.

and outside the geometrical phantom; the results of our modified EM algorithm are shown in Fig. 7. Figure 7 (c - e) show the results of our segmentation for the geometrical phantom at different signal to noise ratios and the errors are calculated with respect to the ground truth from the phantom.

\section{Conclusion}

We have presented an approach for image segmentation which depends on both the intensity gray level and the shape information, and we have applied the algorithm on 2D and 3D images. Apart from the other shape based segmentation methods which calculate the mean and the variance and assume gaussian distribution, in our method, the mean and variance are all embedded into the estimated density, which is calculated using our modified EM algorithm. This algorithm is notably fast, and works both in 2D and 3D.

\section{References}

1. M. Rousson and N. Paragios, "Shape priors for level set representations," Proc. European Conf. on Computer Vision, Vol. 2351 of Lecture Notes in Computer Science, Springer, 2002. 
2. M. Leventon, W. L. Grimson, and O. Faugeras, "Statistical shape influence in geodesic active contours," IEEE CVPR, 2000, pp. 1316-1324.

3. Y. Chen, S. Thiruvenkadam, H. Tagare, F. Huang, D. Wilson, "On the incorporation of shape priors into geometric active contours," IEEE VLSM, pp. 145-152, 2001 .

4. A. Tsai, A.J. Yezzi, W.M. Wells et al., "Model-Based Curve Evolution Technique for Image Segmentation," IEEE CVPR, 2001, pp. 463-468.

5. N. Paragios, "A level set approach for shape-driven segmentation and tracking of the left ventricle," IEEE Trans. on Medical Imaging, vol. 22 , pp. 773-776 , 2003.

6. A. Litvin and W.C. Karl, "Levelset based segmentation using data driven shape prior on feature histograms," IEEE Workshop on Statistical Signal Processing, pp.166-169, 2003.

7. A. Tsai, W. Wells, C. Tempany, E. Grimson and A. Willsky, "Mutual Information in Coupled Multi-Shape Model for Medical Image Segmentation," Medical Image Analysis, Vol. 8, No.4, pp. 429-445, 2004.

8. J. Yang, and J. Duncan, "3D image segmentation of deformable objects with joint shape-intensity prior models using level sets," Medical Image Analysis, Vol. 8, pp. 285-294, 2004.

9. M. Kass, A. Witkin, and D. Terzopoulos, "Snakes: Active contour models," International Journal of Computer Vision, vol. 1, pp. 321-331, 1987.

10. P. Viola and W.M. Wells, "Alignment by maximization of mutual information," In Proc. 5th Int. Conf. Computer Vision, 1995, pp. 16-23.

11. A.A. Farag, A. El-Baz, and G. Gimel'farb "Density Estimation Using Modified Expectation Maximization for a linear combination of Gaussians, IEEE ICIP, 2004, Vol. I, pp. 194-197.

12. H. Takizawa, K. Sugiura, M. Baba et al., "Analysis of intracerebral hematoma shapes by numerical computer simulation using the finite element method," Neurol. Med. Chir., Vol. 34, pp. 65-69, 1994. 